\title{
GISAXS and GIWAXS analysis of amorphous-nanocrystalline silicon thin films
}

K. Juraić(a), D. Gracin(a), B. Šantić(a), D. Meljanac(a), N. Zorić(a), A. Gajović(a), P. Dubček(a),

\section{S. Bernstorff(b), M. Čeh(c)}

(a) Ruđer Bošković Institute, Bijenička c.10000 Zagreb, Croatia

(b) Sincrotrone Trieste, SS $14 \mathrm{~km}$ 163.5, 34012 Basovizza (TS), Italy

(c)Jožef Stefan Institute, Jamova 39, 1000 Ljubljana, Slovenia

\begin{abstract}
Amorphous-nanocrystalline silicon thin films were deposited by Plasma Enhanced Chemical Vapor Deposition (PECVD) on glass substrate with various silicon nanocrystal size distributions and volume fractions. The samples were examined by Grazing Incidence Small Angle X-ray Scattering (GISAXS) and Grazing Incidence Wide Angle X-ray Scattering (GIWAXS) at the Austrian SAXS beamline (Synchrotron Elettra, Trieste) using an X-ray beam energy of $8 \mathrm{keV}$. The grazing incidence angle varied from the critical angle to $0.2 \mathrm{deg}$. above the critical angle. This allowed the examination of the samples at different depths, and the distinction of the surface scattering contribution from the particles scattering in the bulk. The sizes of the "particles" obtained from the horizontal and vertical sections of 2D GISAXS patterns were between 2 and 6 nanometers. Since GISAXS is sensitive to electron density differences (contrast) between the scattering bodies and the surrounding matrix, it is not evident whether the particles are nanocrystals or just voids embedded in amorphous matrix. However, the size of the crystals calculated from the line-shape analysis of peaks in GIWAXS spectra and the crystal size distribution obtained from High-Resolution Transmission Electron Microscopy (HRTEM) images agree well with the size of "particles" estimated from GISAXS, strongly indicating that the observed particles are silicon nanocrystals.
\end{abstract}




\section{Introduction}

Nanocrystalline silicon in form of layers with thickness of ten to several hundred nanometers attracts attention as a possible candidate for high efficient thin film solar cells that use quantum confinement effects present due to small dimensions of crystals. The key structural objects that are expected to enable high efficiency are nano-meter sized objects in the form of ordered 3D domains, quantum dots, QD, called also nano-crystals. The optical, electrical and vibrational properties of QD in such films are not yet fully explained, among others due to the substantial influence of matrix effects. The high influence of the close neighborhood comes due to the small dimensions of the QD which results in a large surface-to-volume ratio [1-3].

Therefore it is important to measure nano-structural properties of thin films with embedded nano-crystals in a way that provides information about the crystals and the properties of their close surrounding. For that purpose, we tested the possibility of using grazing incidence small angle x-ray scattering (GISAXS) [4] to estimate the individual size and shape of inhomogeneities in thin film. Grazing incidence wide angle x-ray scattering (GIWAXS), measured simultaneously, was used to identify the nature of the particles. This was expected to enable distinguishing voids from ordered domains. The line width in GIWAXS spectra also provides additional information about the size of the nanocrystals. The depth profiling of films was done by variation of the grazing incidence angle. The various nano-structural units were identified by proper computer modeling combined with microscopic images [5-10].

\section{Experimental}

Six nanocrystalline silicon thin film samples were deposited on glass substrates using plasma enhanced chemical vapor deposition (PECVD). The plasma was created by radio-frequency excitation while the working gas was a mixture of $\mathrm{SiH}_{4}$ and $\mathrm{H}_{2}(92-95 \%)$. A variation in the nanocrystal size and size distribution was obtained by varying the discharge power and hydrogen to silane ratio, as described previously [11-12]. The thickness of the films estimated from 
transmittance measurements were in the range $200-500 \mathrm{~nm}$ while the crystalline to amorphous volume ratio varied from 10 to some $40 \%$. The crystallinity was estimated as the ratio between corresponding areas of amorphous and crystalline TO phonon peaks in Raman spectra [3]. GISAXS and GIWAXS experiments were performed at the Austrian SAXS beamline at the synchrotron Elettra (Trieste, Italy) [13]. The energy of the synchrotron x-ray radiation was $8 \mathrm{keV}$ $(0.154 \mathrm{~nm})$. The samples were mounted on a sample holder which could be rotated computer controlled in steps of $10^{-3} \mathrm{deg}$.

GISAXS and GIWAXS patterns were collected simultaneously with two different detectors. GISAXS images (patterns) were collected by a two dimensional CCD camera with 1024 x 1024 pixels, positioned in the plane perpendicular to the incident $\mathrm{x}$-ray beam, at a distance of $2 \mathrm{~m}$ from the sample holder. For better statistics, GISAXS images were taken as an average of 64 exposures in a series. In front of the central part of the CCD detector a beam stop was inserted to avoid detector damage by the intense direct (specular) x-ray beam.

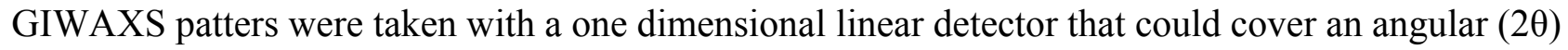
range from 20 to $60 \mathrm{deg}$.

For each sample, GISAXS/GIWAXS pattern were recorded at an angle of incidence equal to the critical angle for that sample $\left(\alpha_{C}\right)$. For crystalline silicon a critical angle of $\alpha_{c}=0.22 \mathrm{deg}$ is found for $8 \mathrm{keV}$ photon energy. Subsequently, the angle of incidence was increased in five steps $\left(\alpha_{\mathrm{c}}+0.02\right.$ deg., $\alpha_{c}+0.05$ deg., $\alpha_{c}+0.10$ deg., $\alpha_{c}+0.15$ deg., $\alpha_{c}+0.20$ deg.). These values of the incident angles correspond approximately to values of the penetration depth of $50 \mathrm{~nm}, 90 \mathrm{~nm}, 140 \mathrm{~nm}, 180 \mathrm{~nm}$ and $220 \mathrm{~nm}$ assuming an absorption coefficient as for crystalline silicon.

Before numerical analysis, the GISAXS/GIWAXS patterns were corrected for the detector response, and the refraction effect at the air-sample boundary.

HRTEM were performed on a Philips CM200 FEG microscope with a field emission gun operating at $200 \mathrm{kV}$. The samples were polished and thinned by an ion beam for transmission electron microscopy. 


\section{Results and discussion}

The typical nano-structure of deposited films is characterized by nano-sized ordered domains, from $2 \mathrm{~nm}$ up to some $8 \mathrm{~nm}$, embedded in amorphous matrix, as is illustrated by the HRTEM micrograph given in Fig. 1. The crystals have a broad size distribution that was estimated from HRTEM micrographs using the software package Uthscsa ImageTool for Windows 3.00, 1995-2002 for crystallite area measurements. The typical result for the sample with $30 \%$ crystal volume contribution is represented by the histogram shown in Fig. 2. Here the lines represent mathematical fits to the measurements using log-normal distribution. In order to estimate the volume contribution for each crystal size, the size distribution is multiplied by the corresponding volume and divided by the total volume of the sample (blue (right) line and right axis in Fig. 2). The maximum of the crystal sizes distribution varied from 2 to $8 \mathrm{~nm}$ where higher values corresponded to a higher crystal fraction. With higher crystallinity, the distribution became wider.

The density of this amorphous matrix is usually $5-10 \%$ lower than the density of the crystalline material and usually contains nano-sized voids [15]. Therefore it is not so straight forward that the ordered domains can be seen by GISAXS although all of the samples exhibit a strong GISAXS signal like that in Fig. 3. The $\mathrm{Q}_{z}$-axis represents the scattering perpendicular to the surface while the $\mathrm{Q}_{\mathrm{y}}$-axis corresponds to the direction parallel to the samples surface. The shape of the signal is rounded, but slightly elongated in the direction perpendicular to the sample surface, indicating a rounded shape of the nanoparticles that is slightly flattened in the direction perpendicular to the film surface.

For the purpose of a quantitative analysis, for each value of angle of incidence cross-sections were made in directions parallel and perpendicular to the sample surface, as is indicated in Fig. 3. The vertical section was made out of the specular plane to avoid the beam stop area where the scattered intensity is highly reduced. The horizontal section was made above the Yoneda peak to avoid the 
dominant surface scattering contribution. As an example in Fig. 4 vertical sections for a few different angles of incidence are displayed. The absolute value of the incident angle $\alpha_{\mathrm{i}}$ is determined as half of the specular beam position. For all sections the Yoneda peak is positioned at angles equal to the sum of incident and critical angle $\left(\alpha_{\mathrm{i}}+\alpha_{\mathrm{c}}\right)$. So from its position and known angle of incidence, it is possible to estimate the value for the critical angle. Values for critical angles estimated in that way are very close to the values for crystalline silicon.

From Fig. 4 it can be seen that the slope of the intensity curve for $\mathrm{Q}_{\mathrm{z}}$ values near the Yoneda peak and slightly above is decreasing as the angle of incidence rises, indicating that the larger nanoparticles are placed near the sample surface.

The average particle dimensions (radius of gyration) were calculated for each 1D section in parallel and vertical directions using Guinier's approximation [14] and assuming a spherical shape of the nanoparticles:

$$
I(Q)=(\Delta n)^{2} e^{-Q^{2} R_{G}^{2} / 3}
$$

where $\Delta \mathrm{n}$ is the electron density difference and $\mathrm{R}_{\mathrm{G}}$ is radius of gyration. The radius of gyration is defined as the mean square distance from the centre of gravity, where the role of "mass" is played by the electrons. Guinier's approximation for the scattering intensity is valid for angles where $Q \cdot R<1$, where $\mathrm{R}$ is radius of the scattering object. The radius of gyration can be found simply by plotting $\ln I(Q)$ against $Q^{2}$ (Fig. 5). For a solution of monodisperse particles with the same shape, the plot will show a straight line with slope $-R_{G}^{2} / 3$ for the region up to $Q \cdot R<1$. The results of the Guinier fit are displayed in Fig. 6 as a function of grazing incidence angle. The value at the critical angle reflects mostly the scattering on the samples surface. By increasing the incidence angle, most of the scattering comes from the "bulk" of the sample. The particles dimensions are larger in the direction parallel to the sample surface, as compared to the direction perpendicular to the films surface. Furthermore, the "particles" closer to the surface are larger than the "particles" in the bulk of the films. 
The GIWAXS spectra, recorded simultaneously with GISAXS, correspond to Si crystals (Fig. 7). The characteristic diffraction lines are broadened and their width differs from sample to sample and by variation of the incidence angle, indicating the variation of crystals sizes. The general expression for line broadening is given by Williamson-Hall [16]:

$$
\left(\varepsilon_{\text {obs }}-\varepsilon_{\text {instr }}\right)=\frac{K \lambda}{D_{V} \cos \theta}+4 \varepsilon_{\text {str }} \tan \theta
$$

where $\varepsilon_{\mathrm{obs}}$ is the line broadening, $\varepsilon_{\mathrm{instr}}$ is the instrumental broadening, $\mathrm{K}$ is the Scherrer constant which is strong dependent nof the nanoparticles shape (here is assumed that $K=1$ ), $\lambda$ is the $\mathrm{x}$-ray wavelength, $\mathrm{D}_{\mathrm{v}}$ is the volume size of the nano-crystals and $\varepsilon_{\text {str }}$ is the strain. Assuming that the instrumental broadening and strain can be neglected in comparison to the size broadening, the inverse widths of the diffraction lines are proportional to the particles sizes. For the quantitative analysis, the [111] silicon peak was used. The peak width (FWHM) was calculated by fitting the peak to Voigt profile and the average crystal sizes were calculated using equation (2). The instrumental line broadening was estimated comparing tin-oxide thin film peaks patterns obtained from GIWAXS and from XRD measured using an instrument with a known instrumental line broadening. The estimated value of $0.16 \mathrm{deg}$ for instrumental line broadening is used in equation (2). That value is much lower then the actual line broadening $(2.1-5.5$ deg. $)$

The average crystal sizes estimated from GIWAXS are plotted in Fig. 8 together with the maximum values of the crystal size distribution from HRTEM as a function of ,particle" sizes obtained by GISAXS. For samples with GISAXS particle size greater then $3 \mathrm{~nm}$ the estimated particle size from both GIWAXS and HRTEM measurement are shown, while for samples with GISAXS particle size lower then $3 \mathrm{~nm}$ the particle size was estimated just from a GIWAXS or a HRTEM measurement. The plotted data show a very good agreement assuming that the "particles" in GISAXS are nano-crystals, and that thus GISAXS is a well chosen method for the analysis of the size and size distribution of ordered domains in an amorphous matrix of the same material.

\section{Conclusion}


Thin silicon films containing nano-crystals embedded in an amorphous matrix were analyzed using GISAXS and GIWAXS. The sizes between 2 and $6 \mathrm{~nm}$ observed by GISAXS for the scattering centers correspond to the particles sizes estimated from GIWAXS and measured by HRTEM. This proves that it is possible to distinguish by GISAXS ordered domains (nano-crystals) from their amorphous matrix even if the difference in electron density between them is small.

\section{Acknowledgments}

The authors thank Medaja Gec (Institute Jožef Stefan, Ljubljana, Slovenia) for preparing the crosssectioned samples for the TEM observations. This work was supported by the Croatian Ministry of Science Education and Sport, grants "The thin film silicon on the amorphous to crystalline transition" (098-0982886-2894) and "Physics and applications of nanostructures and bulk matter" (098-0982904-2898); by the National Foundation of Science and High Education, by the European Community through the Integrated Infrastructure Initiative "European Light Sources ActivitiesSynchrotrons and Free Electron lasers" and by the Croatian-Slovenian bilateral project BIHR/0708-028. 


\section{List of references}

[1] T.J. Bukovski., J.H. Simmons, Crit.Rev.Solid State Mat. Sci ., 27 (2002) 119.

[2] M.C. Beard, K.P. Knutsen, P. Yu, J.M. Luther, Q. Song, W.K. Metzger, R.J. Ellingson, A.J. Nozik, NanoLett., 7 (2007) 2506.

[3] D. Gracin, A. Gajović, K. Juraić, M. Čeh, Z. Remeš, A. Poruba, M. Vaneček, J. Non-Cryst. Solids, 354 (2008) 2286.

[4] T. Metzger, V. Favre-Nicolin, G. Renaud, H. Renevier, and T. Schülli "Nanostructures in the light of synchrotron radiation: surface-sensitive X-ray techniques and anomalous scattering." in: Characterization of Semiconductor Heterostructures and Nanostructures. Edited by C. Lamberti, Elsevier (2008).

[5] D.L. Williamson, Mater. Res. Soc. Symp. Proc. 377 (1995) 251.

[6] U.K. Das, J.K. Rath, D.L. Williamson, P. Chaudhuri, Jap. J. Appl. Phys., 39 (2000) 2530.

[7] U.K. Das, A.R. Middya. J.K. Rath, C. Longeaud, D.L. Williamson. P. Chaudhuri, J. NonCryst. Solids., 276 (2001) 46.

[8] J. Shinar., R. Shinar, D.L. Williamson, S. Mitra, H. Kavak, V.L. Dalal, Phys. Rev. B, 60 (1999) 15875.

[9] A.H. Mahan, Y. Xu. D.L. Williamson, W. Beyer, J.D. Perkins, M. Vanecek, L.M. Gedvilas, B.P. Nelson, J. Appl. Phys., 90 (2001) 5038.

[10] S.J. Gurman, B.T. Williams. J.C. Amiss, J. Phys. Condens. Matter. 12 (2000) 5981.

[11] D. Gracin, S. Bernstorff, P. Dubček, A. Gajović, K. Juraić, Thin Solid Films, 515 (2007) 5615 .

[12] D. Gracin, S. Bernstorff, P. Dubcek, A. Gajovic, K. Juraic, J. Appl. Cryst., 40 (2007) 373.

[13] Amenitsch H. Amenitsch, S. Bernstorff, P. Laggner, Rev. Sci. Instrum., 66 (1995) 1624.

[14] O. Glatter, O. Kratky, Small Angle X-ray Scattering, (Academic Press, London, 1982) p. 25.

[15] D. Gracin, K. Juraic, P. Dubcek, A. Gajovic, S. Bernstorff:, App. Surf. Sci. 252 (2006) 5598

[16] J.K. Williamson, W.H. Hall, Acta Metall., 1 (1953) 22. 


\section{Figures captions}

Figure 1 HRTEM image of a sample with average GISAXS dimensions of 4nm.

Figure 2 Size distribution (histogram) of nanocrystals as determined from HRTEM micrographs and fitted to a log-normal distribution (left axis). On the right side (axis) the relative volume distribution of nanocrystals is displayed.

Figure 3 Representative 2D GISAXS image of nanocrystalline silicon thin film for an angle of incidence $\alpha=\alpha_{\mathrm{c}}+0.02^{\circ}$. $\mathrm{Q}_{\mathrm{y}}$ and $\mathrm{Q}_{\mathrm{z}}$ are scattering wave vectors parallel and perpendicular to the sample surface. The straight lines represent the positions of the horizontal and the vertical sections taken for the Guinier analysis.

Figure 4 One dimensional intensity distribution vs. scattering wave vector for vertical sections of 2D GISAXS images taken along the lines in Fig. 3, for different angles of incidence. The Yoneda peak position is indicated.

Figure 5 Guinier plots (logarithm of intensity vs. the square of the scattering wave vector) for the sections presented in Fig.4. The full lines represent the best linear fits. The gradients were used to determine the radii of gyration, $\mathrm{R}_{\mathrm{G}}$.

Figure 6 Radius of gyration as a function of the angle of incidence for horizontal $(\mathrm{H})$ and vertical (V) sections. In the inset the shape of particles is indicated.

Figure 7 Silicon peak near $28 \mathrm{deg}$. in the GIWAXS pattern as a function of the angle of incidence. For higher angles of incidence the silicon peak is wider.

Figure 8 Comparison of the average crystal sizes estimated for GIWAXS ( $\mathrm{d}_{\text {GIWAXS }}$ ) and crystal sizes estimated from HRTEM ( $\left.\mathrm{d}_{\text {HRTEM}}\right)$ with average sizes of "particles" estimated from GISAXS. The straight line is an eye guide. 


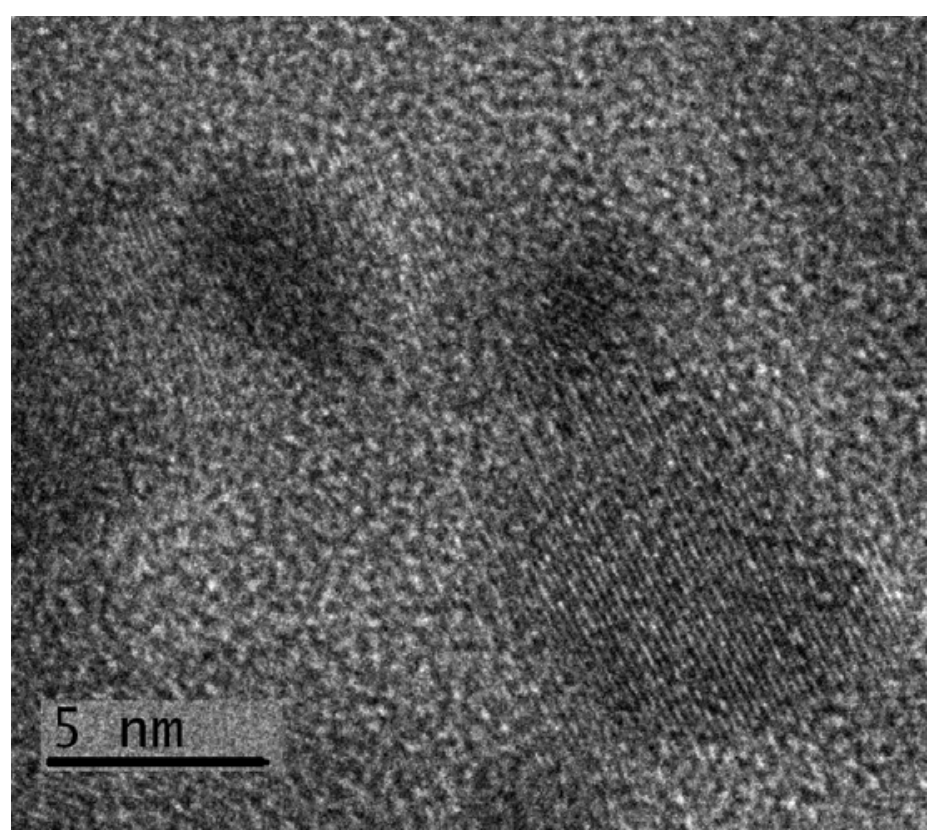

Figure 1 


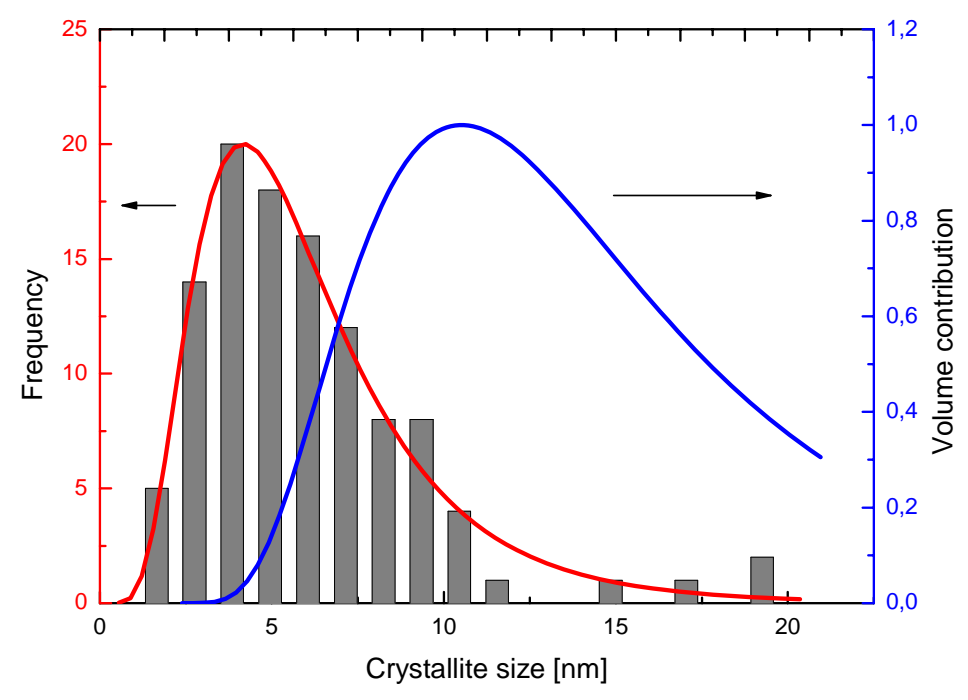

Figure 2 


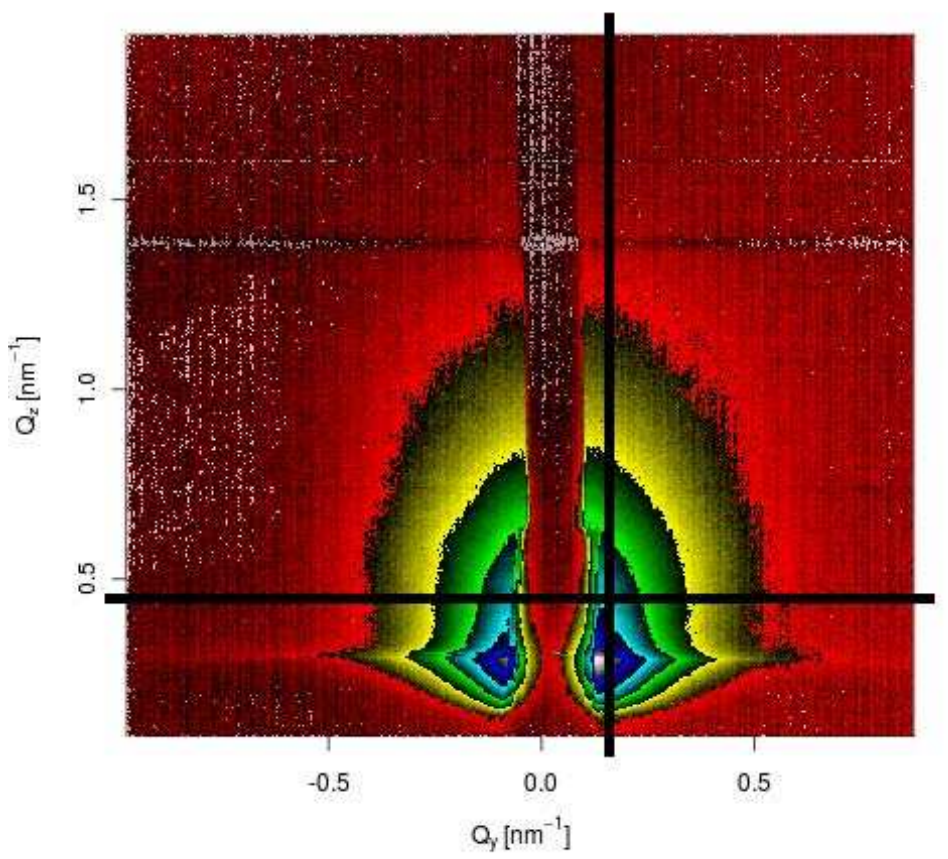

Figure 3 


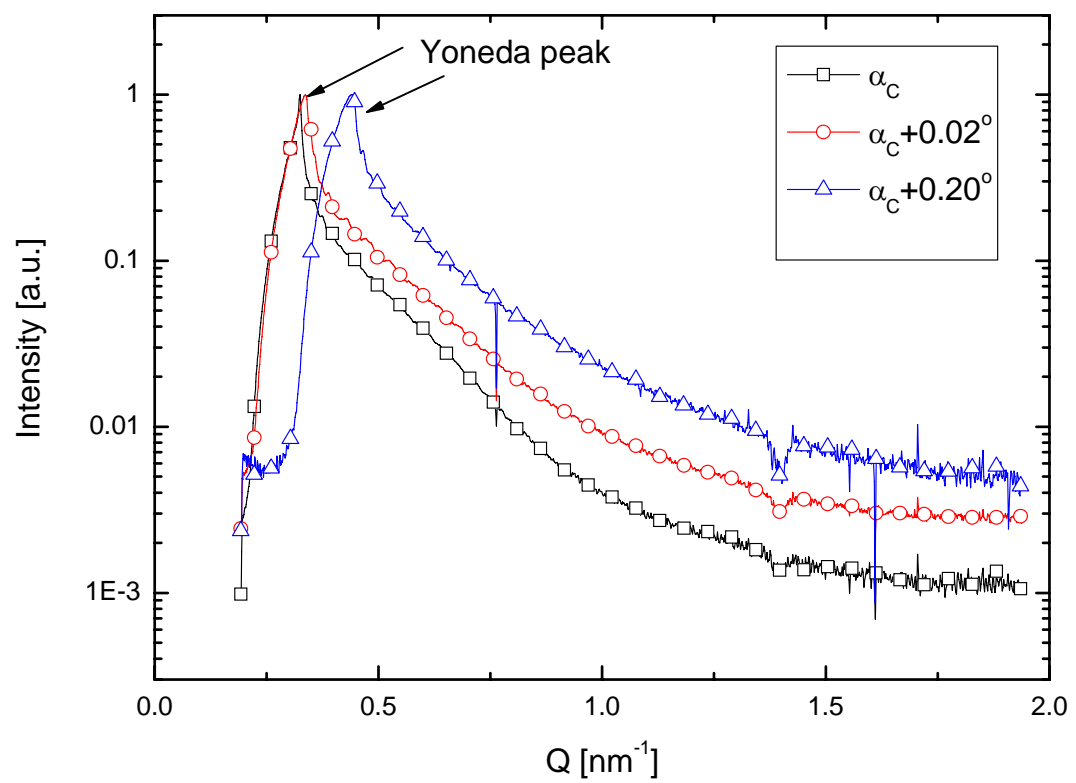

Figure 4 


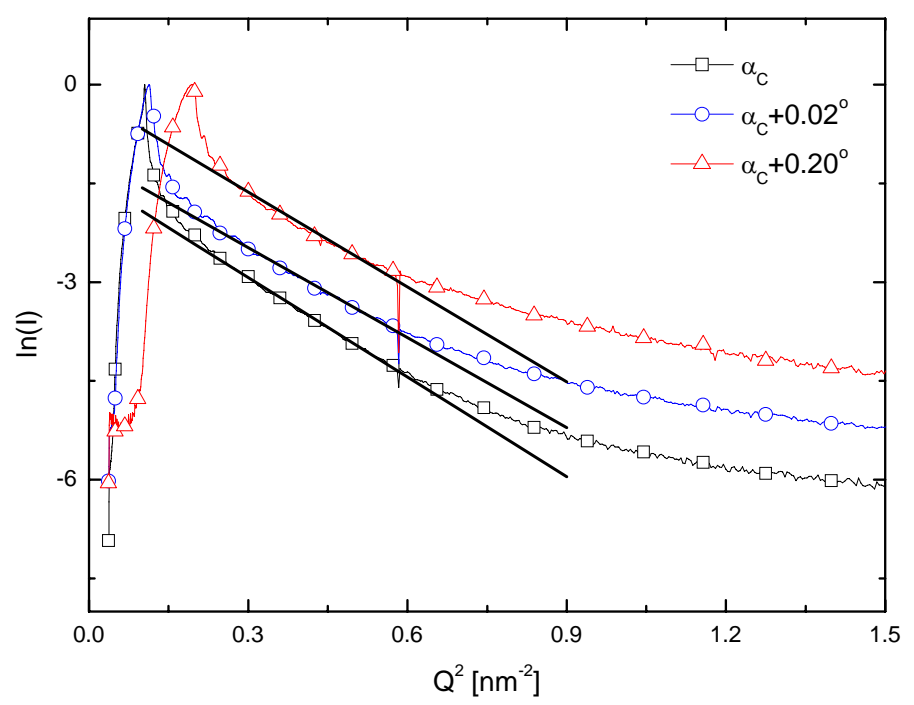

Figure 5 


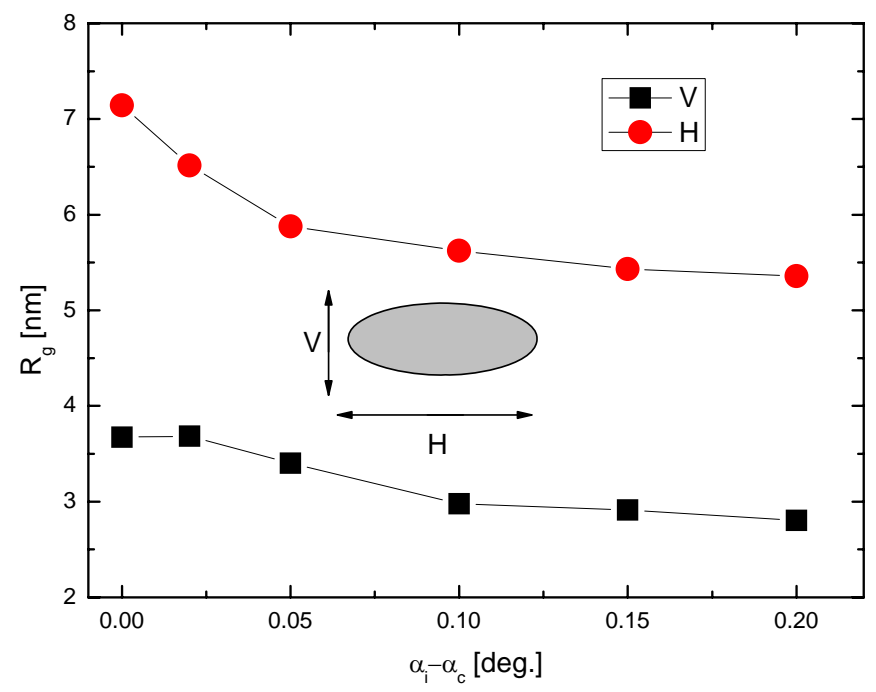

Figure 6 


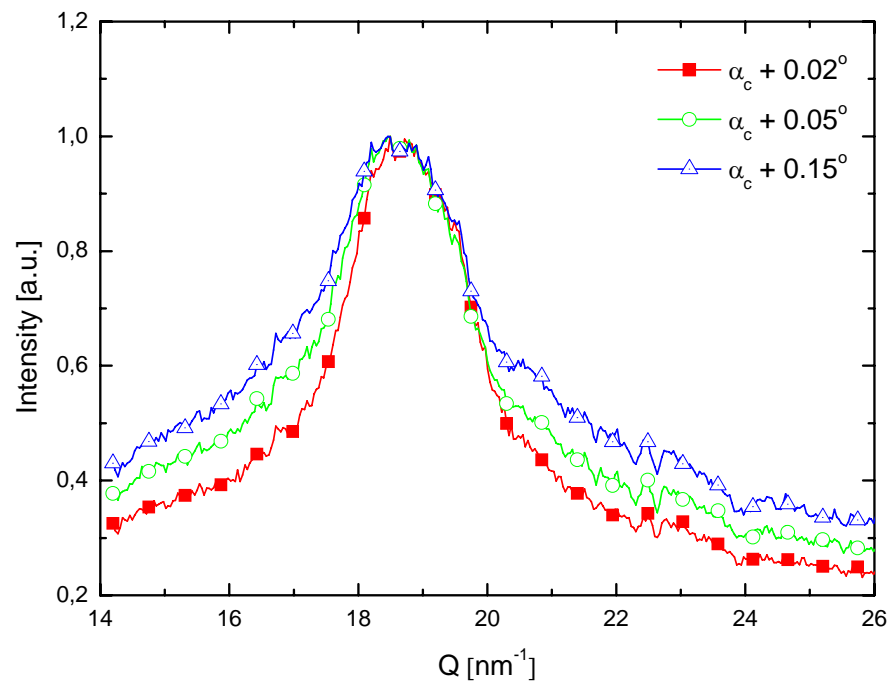

Figure 7 


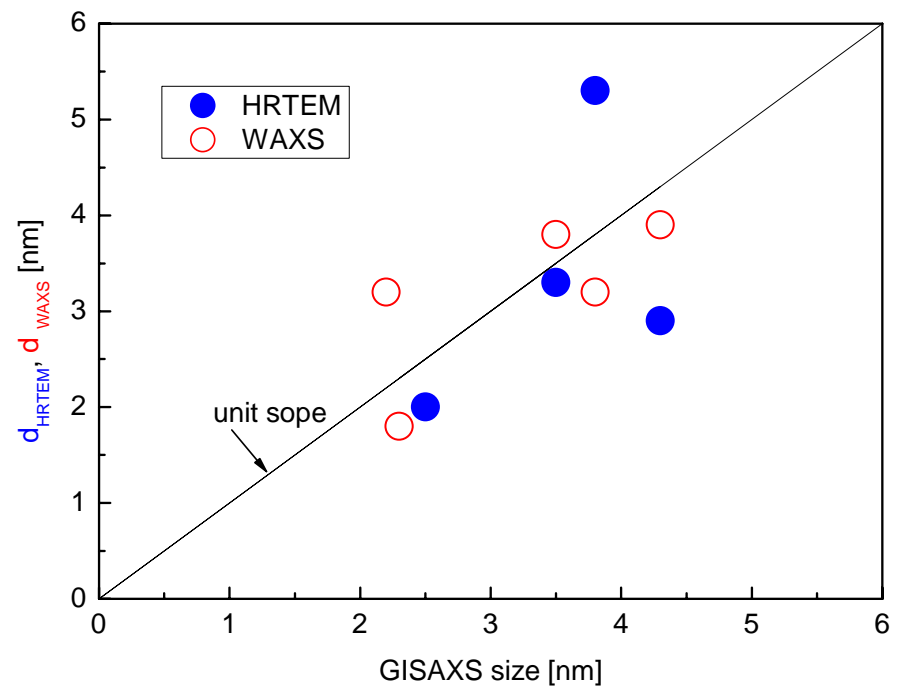

Figure 8 